\title{
A COMPARATIVE STUDY IN WIRELESS SENSOR NETWORKS
}

1 Hasan Al-Refai

1 Department of Computer Information Systems, Philadelphia University, Amman Jordan 2Department of Management Information Systems, Philadelphia University, Amman

Jordan

3Dean of IT, Philadelphia University, Amman Jordan

\begin{abstract}
Sensor networks consist of a large number of small, low-powered wireless nodes with limited computation, communication, and sensing abilities, in a battery-powered sensor network, energy and communication bandwidth are a precious resources. Thus, there is a need to adapt the networking process to match the application in order to minimize the resources consumed and extend the life of the network. In this paper, we introduce a comparative study in different routing algorithms that propose vital solutions to the most important issues that should be taken into account when designing wireless network which are reliability, lifetime, communication bandwidth, transmission rand, and finally the limited energy issue, so we will introduce their algorithms and discuss how did they propose to solve such of these challenges and finally we will do some evaluation to each approach.
\end{abstract}

\section{KEYWORDS}

Wireless sensor networks; Geographic routing; Localized routing; Power-aware routing, reliability, timeliness, query processing, redundancy, energy conservation, QoS.

\section{INTRODUCTION}

Recent advances in technology have made low-cost, low-power wireless sensors a reality. Sensor networks formed from such sensors can be deployed in an ad hoc fashion and cooperate to sense and process a physical phenomenon. As each sensor has a finite battery source, an important feature of sensor network is energy efficiency to extend the network's lifetime.

Thus, the unique characteristics and constraints present many new challenges for the development and application of WSNs. Due to the severe energy constraints of large number of densely deployed sensor nodes, it requires a suite of network protocols to implement various network control and management functions such as synchronization, node localization, and network security.

There are a common routing protocols that have several shortcomings when applied to WSNs, which are mainly due to the energy- constrained nature of such networks [3]. In most cases it is very difficult and even impossible to change or recharge batteries for the sensor nodes. WSNs are characterized with denser levels of sensor nodes deployment, higher unreliability of sensor nodes, and sever power, computation, and memory constraints.

In [1] the authors have proposed an algorithm that investigate how to make on-demand routing protocols adapt quickly to topology changes, this problem is important because such protocols use DOI : $10.5121 /$ ijwmn.2014.6105 
route caches to make routing decisions; it is challenging because topology changes are frequent. They propose proactively disseminating the broken link information to the nodes that have that link in their caches. Proactive cache updating is key in making route caches adapt quickly to topology changes, it is also important to inform only the nodes that have cached a broken link to avoid unnecessary overhead. Thus, when a link failure is detected, the goal is to notify all reachable nodes that have cached the link about the link failure. They define a new cache structure called a cache table to maintain the information necessary for cache updates. A cache table has no capacity limit; its size increases as new routes are discovered and decreases as stale routes are removed.

The algorithm is fully-distributed, adaptive to the topology changes and fault-tolerant, since there is always a way to handle broken link or node failure.

Moreover, this algorithm eliminates the overhead of flooding route request into the network and can construct the multipaths on the fly. Thus, this algorithm is scalable and efficient and it has less communication and storage overhead than other algorithms while providing greater resilience to node failures.

In $[3,9]$ the authors have proposed a fast, randomized, distributed algorithm for organizing the sensors in a wireless sensor network in a hierarchy of clusters with an objective of minimizing the energy spent in communicating the information to the information processing center, but in the other hand, they assume that the communication environment is contention and error-free, so sensors do not have to retransmit any data, in the other words the overall reliability of the system is on a stake, since there is no redundancy mechanism to cope with network failures.

So the main concentration in this approach is how to reduce the overall power consumption in the system to cope with limited battery power or battery constraints.

In $[4,7]$ the authors have proposed an algorithm that handle the fault tolerant and reliability requirements for data information in sensor networks, so in this approach each node has a maximum transmission range to avoid link failure due to the power limitations of sensors(small battery), also it uses Total Time to Live (TTL) to adjust the level of data reliability, so that the more TTL the more possible paths used by the data the more robust it would be in the face of link or node failures, and TTL is always chosen to be slightly higher than the diameter of a network for the sake of necessary redundant packet.

In $[5,8]$ the authors have proposed an algorithm that is very based on query-based sensor systems, in which a user would issue a query with QoS requirements in term of reliability and timeliness and expect a response within a specific time which is called the deadline, where the fault-tolerant for the overall system can be achieved by redundancy of path and source and the optimal path and source redundancy is chosen depends on the routing algorithm they mentioned.

They also determine the optimal redundancy level that could satisfy the QoS requirements while prolonging the lifetime of the wireless sensor network. Since sensor networks are constrained with resources, they develop a hop-by-hop data dissemination paradigm to dynamically form multiple paths for data delivery, instead of incurring extra overhead to first formulate multiple paths before data delivery. 
International Journal of Wireless \& Mobile Networks (IJWMN) Vol. 6, No. 1, February 2014

\section{THE PROPOSED ROUTING ALGORITHM}

In [13] the authors have proposed a dynamic source routing protocol to address the cache staleness issue which limits the mobility of the overall network because of the delay that is, a node is not notified when a cached route breaks until it uses the route to send packets.

When a node detects a link failure, the goal is to notify all reachable nodes that have cached that link to update their caches. To achieve this goal, the node detecting a link failure needs to know which nodes have cached the broken link and needs to notify such nodes efficiently. This goal is very challenging because of mobility and the fast propagation of routing information.

The solution is to keep track of topology propagation state in a distribution manner. Topology propagation state means which node has cached which link. In a cache table, a node not only stores routes but also maintains two types of information for each route which are how well routing information is synchronized among nodes on a route and which neighbor has learned which links through a route reply. Each node gathers such information during route discoveries and data transmission, without introducing additional overhead.

This approach enables dynamic source routing (DSR) to quickly remove stale routes, thus reducing packet losses, delivery latency, and replies.

In $[14,17]$ the authors have proposed the geographical power efficient routing (GPER) protocol where each node knows whereabouts of the nodes within its power range and each intermediate node locally identifies the most power efficient path to the sub destination within its reach and forwards the packet to the next node on this locally identified path. The packet however, may not follow this path exactly since the sub destination changes

from node to node. Two mechanisms, force routing and perimeter routing, are applied in GPER to prevent infinite loops.

So, each sensor node makes local decisions as to how far to transmit; therefore, the protocol is power-efficient, localized, highly distributed, and scalable, in GPER, given a final destination, each node first establishes a sub destination within its maximum radio range, the node, however, may decide to relay the packet to this sub destination through an intermediary node or alter the sub destination if this will preserve power.

Three geographic multipath routing algorithms have been introduced (MGPSR, MGPERsub, and MGPERhop), which generate alternative paths probabilistically based only on local information. These algorithms identify and use different routes for different messages between a source and a destination, they take away from the load on the nodes that are on the preferred path between the end-points and distribute this load on the other nodes that are in the neighborhood. Of course, as these paths are less perfect than the preferred path, they are expected to introduce a degree of overhead, but experiments show that multipath routing improves the lifetime of the network, especially in cases where the communication is non-uniformly distributed in the network

In $[1,20]$ the authors have proposed a distributed, randomized, clustering algorithm to organize the sensors in a wireless sensor network into clusters. Then they extend this algorithm to generate a hierarchy of cluster heads and observe that the energy savings increase with the number of levels in the hierarchy, they assume that sensors transmit at the same power level and hence they all have the same radio range, moreover they suppose that each sensor uses 1 unit of energy to transmit or receive I unit of data and finally, they assume that the network is fault-tolerant and the 
communication environment is contention and error-free and sensor do not have to retransmit any data.

A routing infrastructure has been used in this approach, hence when a sensor communicates data to another sensor, only sensors on the routing path forward the data, which means the route should be established first and then data can be transmitted which adds time overhead to the system at all.

The algorithm states that each sensor in network becomes a cluster head and advertises itself to the sensors within its radio range, and it is called the volunteer cluster head, this advertisement is forwarded to all the sensors that are no more than $\mathrm{k}$ hops away from the cluster head where $\mathrm{k}$ is chosen by the algorithm and any sensor that receives such advertisements and is not itself a cluster head joins the cluster of the closest cluster head, any sensor that is neither a cluster head no has joined any cluster itself becomes a cluster head and then it is called the forced cluster head and since this algorithm is a distributed one, there is no need for clock synchronization between the sensors at all even if they were in the same cluster. Since this algorithm is a hierarchy based then each level does the same and elects the cluster head in the same manner.

In $[15,18]$ the authors have proposed an algorithm based on majority voting in sensor network, under the assumptions that the topology of the network is fixed, each node has a maximum transmission range to overcome the power limitations of sensors, the propagation delay on each functioning link is equivalent, and the processing delay in each node is non zero.

In this approach, when nodes are first deployed, the sink node sends a control packet informing each node of the value of TTL (total time to live) where TTL is chosen under a certain criterion that is, the more TTL the more possible paths used by the data the more robust to cope with link or node failures, on the other hand, TTL Is always taken to be equal to the diameter of the network to eliminate redundant traffic while broadcasting, but for the sake of necessary redundant packet to perform majority voting, TTL should be set to be slightly higher the diameter. The majority voting could be trigger whenever 3 packets with the same sequence number arrive.

In $[16,19]$ the authors have proposed an algorithm based on a hop-by-hop data dissemination paradigm to dynamically form multiple paths for data delivery, instead of incurring extra overhead to first formulate multiple paths before data delivery. They assume that sensors have the same initial energy level and sensors group themselves into distinct clusters in the system for energy conservation purpose, each cluster has a cluster head $(\mathrm{CH})$ which is responsible for data reading and aggregating from sensor within same cluster and the role of cluster head is rotated fairly among sensors in same cluster using a specific algorithm such as HEED or LEACH which will help to conserve energy and prolong the lifetime of the network so that sensors will not quickly deplete their energy, not only that but also the transmission power of a sensor even when it is a cluster head is reduced to a minimum level to enable it to communicate within one-hop radio range, thus a sensor node should use a multi-hop route protocol to enable it to communicate with another sensor distant away.

Two forms of redundancy are used in this approach to help fault tolerance and lifetime reasons which are path and source redundancy, that is, instead of having a single path to connect a source cluster to the processing center, $\mathrm{m}$ disjoint paths may be used, on the other hand, they use $\mathrm{m}$ sensor nodes to return readings to cope with sensor hardware and software faults.

In cluster network a source cluster head must send data information to the processing center and this will consume energy depends on the length of the path between $\mathrm{CH}$ and the processing cluster, if the overall system energy falls below a threshold value say threshold energy then the system is considered as having depleted its energy. That is, the consumed energy can be 
International Journal of Wireless \& Mobile Networks (IJWMN) Vol. 6, No. 1, February 2014

computed in terms of the energy to run the transmitter and receiver circuitry, the amplification energy that is used to achieve acceptable signal to noise ratio, the loss energy due to channel transmission.

Thus, the system lifetime can be defined here as the total number of queries the system can answer correctly until it fails due to either channel or sensor faults or when the system energy approaches the threshold level energy.

In [23] their work based on [21] to conduct a measurement study on a large-scale operating sensor network system, GreenOrbs, with up to 330 nodes deployed in the wild. they aim to understand how the sensor network performs when it scales to contain hundreds or even thousands of nodes.

\section{EVALUATION}

The approach in [13] achieves fault-tolerance to the wireless network against link or node failures by using caching technique in dynamic source routing which quickly removes stale routes, thus it reduce packet losses, delivery latency, and replies.

The approach in $[14,17]$ achieves power efficient routing method which enables the nodes to adaptively chooses the transmission range to minimize the power consumption of the sensor as well as of the non-participating listeners, and by using three families of probabilistic geographical multipath routing protocols, they improve the network lifetime of wireless network, in addition, all of these protocols are highly power efficient, distributed and scalable, on the other hand, the reliability of the network still not handled in this approach.

The approach in $[1,19]$ achieves conservation to the total energy by minimizing the total energy spent in the system to communicate the information gathered by these sensors to the information processing center and even this approach has a significant improvement on a time complexity over many clustering algorithms and this makes this approach suitable for networks of large number of nodes, it is still has a problem with reliability since it states that the communication environment is contention and error free, moreover it neglects the fact that the cluster heads in this architecture spend relatively more energy than other sensors because they have to receive information from all the sensors within their clusters, aggregate this information and then communicate to the higher level cluster heads or the information processing center.

The approach in $[15,18]$ achieves reliability and fault tolerance for a simple network with fixed topology which is free of crash failures but it is still unable to cover dynamic and sophisticated networks reliabilities which are changing very quickly, on the other hand it, didn't handle the problem of power limitations of sensors or manage energy usage.

The approach in $[16,19]$ achieves quality of service requirements in terms of reliability and timeliness while maximizing the lifetime of query-based sensor networks by using hop-by-hop packet broadcasting protocol, in the other hand this approach is based on the average behavior without considering dynamics such as more energy may be consumed by some sensors over others or some sensors may fail earlier than others. It doesn't handle multiple QoS levels from multiple queries and the bandwidth here is a bottle-neck, since much redundancy is achieved by hop-by-hope packet broadcasting protocol.

The approach in [23] achieves two contributions, first, they argue that they are the first to conduct a long term and large-scale measurement study on an operating sensor network in the wild. Where they present observations across a variety of layers in the network that provide research 
community empirical experiences on how practical problems affect when the sensor network scales.

Second, based on their basic findings from the system measurement, they propose and initially attempt to validate three conjectures that provide guidelines for future algorithm and protocol designs with larger scale sensor networks. That is, 1- they thought it possible that some of the intermediate nodes bottleneck the entire network, and most of currently used indicators may not accurately capture them; 2- most of the wireless links in large scale sensor networks are physically stable. But due to the dynamic features mainly come from the inherent concurrency of network operations that need to be further investigated and considered in designing scalable network protocols. 3- the environment, while with insignificant dynamics, has an unpredictable impact on thesensor network under it. They suggest that an event based routing structure can be trained optimized and thus better adapt to the wild environment when building a large-scale sensor network.

\section{REFERENCES}

[1] Seema Bandyopadhyay and Edward J, Coyle, An Energy Efficient Hierarchical Clustering Algorithm for Wireless Sensor Networks.

[2] D.B. Johnson, D.A. Maltz, Dynamic source routing in ad hoc wireless networks, in: Mobile Computing, 1996, pp. 153-181.

[3] S. Murthy, J.J. Garcia-Luna-Aceves, An efficient routing protocol for wireless networks, Mobile Networks and Applications 1 (2) (1996) 183-197.

[4] V.D. Park, M.S. Corson, A highly adaptive distributed routing algorithm for mobile wireless networks, in: INFOCOM, 1997, pp. 1405-1413.

[5] C. Perkins, Ad hoc on-demand distance vector routing, in: MILCOM '97 Panel on Ad Hoc Networks, 1997.

[6] C. Perkins, P. Bhagwat, Highly dynamic destinationsequenced distance-vector routing (DSDV) for mobile computers, in: SIGCOMM, 1994.

[7] A. Rao, S. Ratnasamy, C. Papadimitriou, S. Shenker, I. Stoica, Geographic routing without location information, in: MobiCom, 2003, pp. 96-108.

[8] V. Rodoplu, T. Meng, Minimum energy mobile wireless networks, in: ICC, 1998, pp. 1633-1639.

[9] L. Li, J. Halpern, Minimum energy mobile wireless networks revisited, in: ICC, 2001.

[10] Q. Li, J. Aslam, D. Rus, Distributed energy-conserving routing protocols for sensor networks, in: IEEE HICSS, 2002

[11] J.H. Chang, L. Tassiulas, Energy conserving routing in wireless ad hoc networks, in: INFOCOM, 2000, pp. 22-31.

[12] Y.B. Ko, N.H. Vaidya, Location-aided routing (LAR) in mobile ad hoc networks, in: MobiCom, 1998, pp. 66-75.

[13] Xin Yn, Distributed cache updating for the dynamic source routing protocol, IEEE TRANSACTIONS ON MOBILE COMPUTING, VOL. 5, NO. 6, JUNE 2006

[14] Shibo Wu, K. Selc, uk Candan, Power-aware single and multipath geographic routing in sensor networks. Elsevier, Ad Hoc Networks 5 (2007) 974-997

[15] Jen-Yeu Chen, Yen-Shiang Shue, Hakeem Ogunleye, Saurabh Bagchi, A comparative study on data fault tolerant requirements for data propagation in sensor networks.

[16] Anh Phan Speer Ing-Ray Chen Virginia Tech, On Optimal Path and Source Redundancy for Achieving QoS AND Maximizing Lifetime of Query-Based Wireless Sensor Networks

[17] Haojun Huang*, Guangmin Hu, Fucai Yu. Energy-aware multipath geographic routing for detouring mode in wireless sensor networks. European Transactions on Telecommunications. Volume 22, Issue 7, pages 375-387, November 2011

[18] Ehsan, S. A Survey on Energy-Efficient Routing Techniques with QoS Assurances for Wireless Multimedia Sensor Networks. Communications Surveys \& Tutorials, IEEE. Second Quarter 2012

[19] Debnath Bhattacharyya , Tai-hoon Kim , and Subhajit Pal. A Comparative Study of Wireless Sensor Networks and Their Routing Protocols. Journal of MDPI Sensors. 2010. 
International Journal of Wireless \& Mobile Networks (IJWMN) Vol. 6, No. 1, February 2014

[20] Guangjie Han, Lei Shu, Jianhua Ma, Jong Hyuk Park, Jianjun Ni. Power-Aware and Reliable Sensor Selection Based on Trust for Wireless Sensor Networks. Journal of Communications, Vol 5, No 1 (2010)

[21] T. He, P. Vicaire, T. Yan, Q. Cao, G. Zhou, L. Gu, L. Luo, R. Stoleru, J. A. Stankovic, and T. F. Abdelzaher, "Achieving Long-Term Surveillance in VigilNet," TOSN, vol. 5, no. 1, pp. 1-39, 2009.

[22] Shio Kumar Singh, M P Singh, and D K Singh, "Routing Protocols in Wireless Sensor Networks -A Survey", International Journal of Computer Science \& Engineering Survey (IJCSES), November 2010.

[23] Does Wireless Sensor Network Scale? A Measurement Study on GreenOrbs Yunhao Liu, Yuan He1, Mo Li, Jiliang Wang1, Kebin Liu, Lufeng Mo, Wei Dong, Zheng Yang, Min Xi, Jizhong Zhao, Xiang-Yang Li. 2013 Zhejiang University. Singapore.

\section{AUTHORS}

\section{Ass. Prof. Dr. Hasan Alrefai}

Dr. Hasan Al-Refai is an assistant professor and a head of Computer Information Systems department for three years 2009 to 2012 at Philadelphia University, Jordan. He got his $\mathrm{PhD}$ from National University of Malaysia, 2005. He joined Philadelphia in the fall (first) semester 2006 after one year e xperience as assistant professor at Yarmouk University, Jordan. His research interests include Cryptography, Mobile Cryptographic protocols, E-

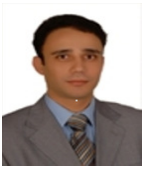

Commerce Security, Formal Methods, Multimedia, Mobile \& Distributed Computing. He has written a number of journal articles and conference papers. He is a member of IJOPCM Editorial Board, International Journal of Open Problems in Computer Science, Program Committee of the Third International Symposium on Innovation in Information \& Communication Technology - ISIICT 2009 (From 15 - 17 December, 2009) Jordan. Reviewer at Third International Symposium on Innovation in Information \& Communication Technology - ISIICT 2009 (From 15 - 17 December, 2009), Member of the steering committee of the fourth International Symposium on Innovation in Information \& Communication Technology - ISIICT 2011 November, 2011.

\section{Dr. ali Alawwneh}

Dr. ali Alawwneh is an associate professor and a head of Management Information Systems department at Philadelphiad University, Jordan. He got his PhD from Arab Academy for Banking and Financial Sceiences, 2008. his Phd involved a study in ebusiness in which he investigated e-business value creation at Jordan Banking Sector through developing a model named e-TOEECLN. He has written a number of journal articles and conference papers.

\section{Dr. Khaldoun Mohammad Batiha}

Dr. Khaldoun Mohammad Batiha is an associate professor and Dean of Information Technology Faculty at Phialdelphia University, Jordan. Has research interests include Computer Networks and Artificial Intelligence. He has written a number of journal articles and conference papers. He has participated in authoring two books are Computer Skills and Analysis and Design Algorithms.
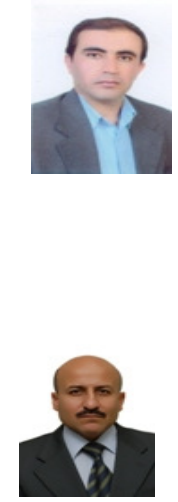